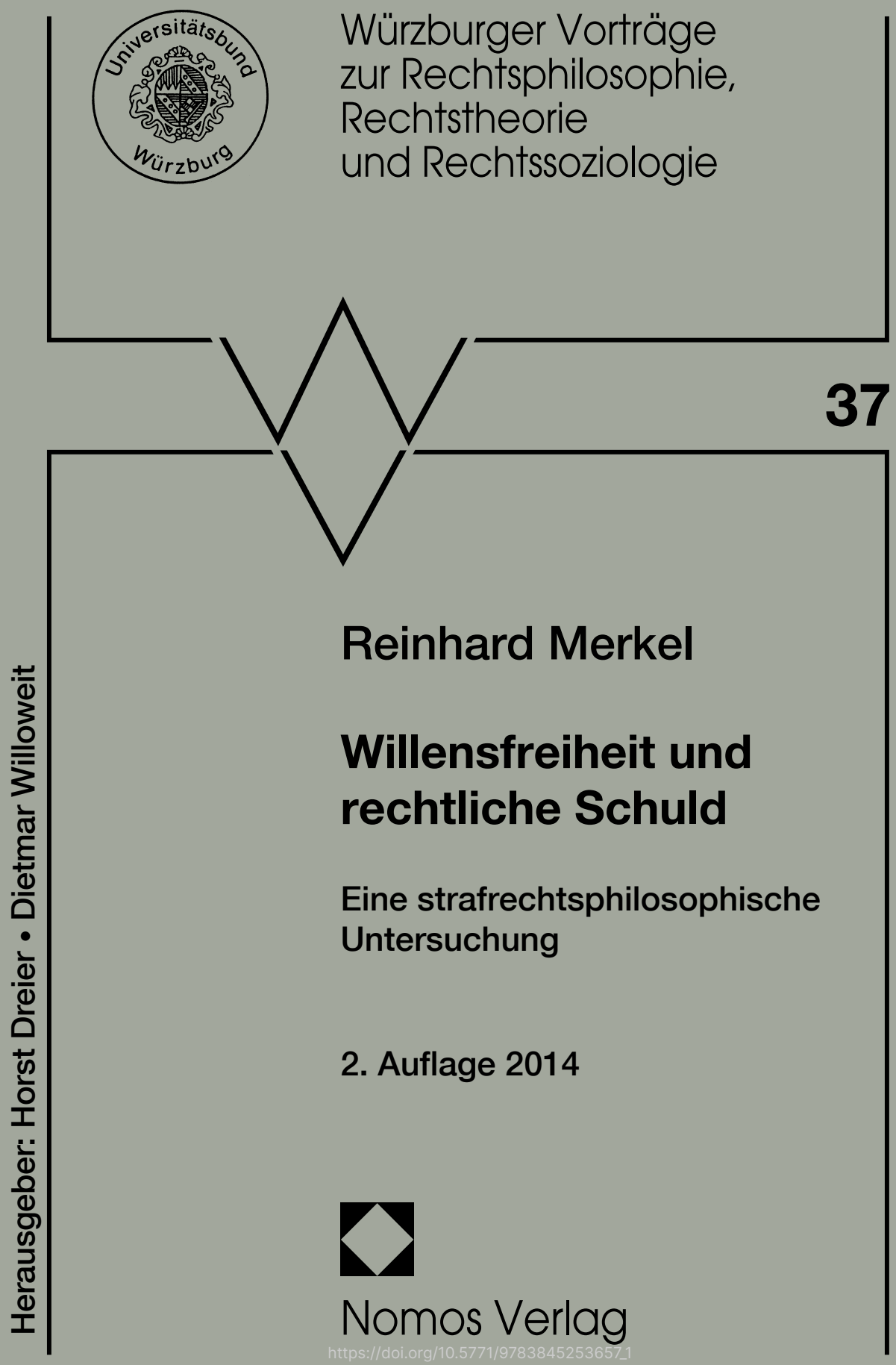


Würzburger Vorträge zur Rechtsphilosophie, Rechtstheorie und Rechtssoziologie

Herausgegeben von Horst Dreier und Dietmar Willoweit

Begründet von Hasso Hofmann, Ulrich Weber ${ }^{\dagger}$ und Edgar Michael Wenz ${ }^{\dagger}$

Heft 37 
Reinhard Merkel

Willensfreiheit und

rechtliche Schuld

Eine strafrechtsphilosophische Untersuchung

2., um ein aktuelles Vorwort ergänzte Auflage 2014 
Vortrag gehalten am 18. Januar 2006

Die Deutsche Nationalbibliothek verzeichnet diese Publikation in der Deutschen Nationalbibliografie; detaillierte bibliografische Daten sind im Internet über http://dnb.d-nb.de abrufbar.

ISBN 978-3-8329-1254-0

2., um ein aktuelles Vorwort ergänzte Auflage 2014

(c) Nomos Verlagsgesellschaft, Baden-Baden 2014. Printed in Germany. Alle Rechte, auch die des Nachdrucks von Auszügen, der fotomechanischen Wiedergabe und der Übersetzung, vorbehalten. Gedruckt auf alterungsbeständigem Papier. 
Für B.

forever 


\section{Vorwort zur 2. Auflage}

Keimzelle dieses kleinen Büchleins war der Vortrag, dessen Datum die Rückseite seines Titelblatts anzeigt und dem es die Aufnahme in die Reihe der „Würzburger Vorträge“ verdankt. Aber so klein ist das Büchlein wieder nicht, um nicht sofort erkennen zu lassen, dass sein Inhalt schon an schierer Quantität über das hinausgeht, was allenfalls in einem Vortrag Platz hätte. In den zwei Jahren zwischen jenem Vortrag und dem Erscheinen der 1. Auflage dieser Abhandlung erweiterte sich deren Gegenstand beträchtlich. Zugleich fügte er sich ein in ein weit umfangreicheres Forschungsprojekt, das mir durch eine großzügige Förderung im Rahmen des Programms „Pro Geisteswissenschaften“ seitens der Fritz Thyssen Stiftung ermöglicht wurde. Es befasst sich mit neuartigen „Interventionen ins Gehirn“ und damit auch ins „Ich“ des Menschen. Dass dazu vor allem manifeste neurotechnische Eingriffe gehören, liegt auf der Hand. Aber sie sind nicht die einzigen. Vielleicht müssen ja auch neue Einsichten dazu gerechnet werden, die uns die Neurowissenschaften zum Verständnis alter metaphysischer Fragen der Philosophie des Geistes liefern mögen, zu deren meisterörterten wiederum das Problem der Willensfreiheit gehört. Auch dessen Lösung, so glauben viele Neurowissenschaftler und manche Philosophen, könnten die empirischen Wissenschaften vom Gehirn möglich machen.

Ich glaube das nicht. Zu meinen verblüffendsten Erfahrungen nach Erscheinen dieses Büchleins gehört die Behauptung mancher Rezensenten, ich erklärte darin die Existenz eines freien Willens für „wissenschaftlich widerlegt". Im Gegenteil! Eines der Anliegen der kleinen Schrift ist es zu zeigen, dass das Problem der Willensfreiheit, was immer man im Einzelnen darunter verstehen mag, ein genuin philosophisches ist und einer naturwissenschaftlichen Lösung schon prinzipiell nicht zugänglich. Selbst wenn man die zahlreichen umstrittenen Unklarheiten in den Begriffen des „Willens“ und der „Freiheit“" (mitsamt ihrem semantischen Umfeld) für behebbar hält, bleiben als Kern des Themas eine Reihe metaphysischer Grundfragen, die sich empirischer Klärung ent- 
ziehen. Das beginnt mit den umstrittenen Prämissen der Diskussion; sie firmieren meist als die Annahmen eines universellen Determinismus oder Indeterminismus und sind ebenfalls schon begrifflich unklar. Es setzt sich fort in den ungelösten Rätseln des klassischen Leib-SeeleProblems, das Schopenhauer den „Weltknoten“ genannt hat. Und es mündet schließlich in dem Problem einer „mentalen Verursachung“ der Frage, wie es kohärent vorstellbar sei, dass körperliche Bewegungen, also Vorgänge der physischen Welt, durch einen „Willen“ ausgelöst werden, der per definitionem zur Sphäre des Nichtphysischen, des „Geistigen“" gehört. Das alles sind Fragen der Philosophie, nicht der Naturwissenschaft. Und ob sie jemals konsensfähig lösbar werden, weiß derzeit niemand. Allerdings ziehen die Naturwissenschaften, insbesondere die Physik, den Lösungsvorschlägen der Metaphysik, die sachlich jenseits ihrer Zuständigkeit liegen, immerhin bestimmte Grenzen: Philosophische Lehren, deren ontologische Implikationen mit den konsentierten Grundannahmen der Physik kollidieren, sind nicht haltbar. Auch das versuche ich zu zeigen.

Damit bin ich beim Hauptanliegen meiner Abhandlung. Betrachtet man die weltweit riesige Menge philosophischer Literatur auch und gerade der jüngeren Vergangenheit zu den angedeuteten Problemen, dann darf man sich ein wenig wundern, dass sie bislang so gut wie kein nennenswertes Echo in der deutschen Strafrechtswissenschaft gefunden hat. Wohl wird dort im Zuge einer ehrwürdigen Tradition nicht selten die (Willens-)Freiheitslehre Immanuel Kants zitiert (wenngleich nach meinem Eindruck meist im Modus der bloßen Paraphrase und regelmäßig nur von gläubigen Kantianern, denen jeder Zweifel an den Argumenten des großen Mannes als unzulässig, ja manchmal geradezu als Sakrileg erscheint). Die weitaus differenzierteren, erheblich tiefer dringenden Analysen der Gegenwartsphilosophie dagegen blieben bislang ohne Würdigung in der Strafrechtsdoktrin, von einer fühlbaren Wirkung auf deren interne Debatten nicht zu reden.

Das im Rahmen des mir Möglichen zu ändern, war das primäre Motiv. Meine Überlegungen wollen deshalb auch verstanden werden als der, wenn ich recht sehe, erste größere Versuch, einige der wichtigsten unter den neueren philosophischen Argumenten zum Problem der Willensfreiheit in die strafrechtliche Diskussion zu importieren. Vor dem Hin- 
tergrund der unüberschaubaren Flut philosophischer Beiträge sind einem solchen Unternehmen naturgemäß enge Grenzen gesetzt. Zwar versuche ich, nicht bloß zu referieren, sondern zu den jeweils dargestellten Streitfragen mit eigenen Erwägungen Farbe zu bekennen. Aber eine weitaus größere Zahl an Argumenten, Modellen und Lösungsvorschlägen, als hier auch nur berührt werden können, bleibt notgedrungen unerwähnt.

Dieser Versuch des Imports philosophischer Analysen ins Strafrecht erzeugt eine leicht erkennbare Asymmetrie zwischen dem philosophischen und dem strafrechtstheoretischen Teil der Abhandlung: Der erstere ist erheblich umfang- und detailreicher als der letztere, dessen Begründungen gelegentlich ein wenig apodiktisch ausfallen. Da ich vor allem Strafrechtswissenschaftler als Leser erwarten durfte, also Kenner der einschlägigen Straftheorien und ihrer Grundlagen, erschien mir das vertretbar. Manche Kommentatoren der Schrift haben es freilich leise tadelnd vermerkt. Ihnen ist zuzugeben, dass die straftheoretischen Konsequenzen, die ich aus der philosophischen Analyse des Freiheitsproblems ziehe, in einem wichtigen Punkt unentwickelt bleiben. Eine „Willensfreiheit", verstanden als die Möglichkeit von Straftätern, sich bei der Entscheidung zur Ausführung ihrer Tat jeweils auch anders zu entscheiden (und dann anders zu handeln), erkläre ich für nicht überzeugend. Als Grundlage der Schuldfähigkeit erscheint sie mir unhaltbar. Das legt die Frage nahe, was dafür an Stelle eines solchen Andershandelnkönnens geeignet wäre. Und hier begnüge ich mich mit dem knappen Hinweis auf ein Modell, das vor allem Claus Roxin seiner Konzeption von Verantwortlichkeit zugrunde legt und mit einem von Peter Noll geprägten Titel überschreibt: „,normative Ansprechbarkeit“. Was dies genau bedeutet, welche begrifflichen Elemente es enthält, was davon empirischer Bestätigung zugänglich ist und was metaphysische Annahme bleibt, und ob es sich schließlich mit dem Wortlaut des § 20 StGB vereinbaren lässt - das alles ist bislang noch kaum eingehend analysiert, geschweige denn bis zur forensischen Verwendbarkeit entwickelt worden. Auch in dieser Abhandlung geschieht das nicht. Drei Jahre nach ihrem Erscheinen habe ich aber einen ersten Versuch dazu unternom- 
men. ${ }^{*}$ Auf ihn mag hier verwiesen werden und der kleinen Schrift das Recht auf die Asymmetrie ihrer beiden theoretischen Grundperspektiven belassen bleiben.

Hamburg im März 2014

Reinhard Merkel

* Merkel, Schuld, Charakter und normative Ansprechbarkeit, in: Heinrich et al. (Hg.), Strafrecht als Scientia Universalis. Festschift für claus Roxin zum 80. Geburtstag, Bd. 1 (2011), S. $7377-761$. 


\section{Inhaltsverzeichnis}

I. Übersicht

II. Grundbegriffe 9

1. Freiheit 9

2. Wille 15

3. Handlungs-/Entscheidungsfreiheit: das geläufige Verständnis 16

III. Zum Verhältnis Determinismus, Freiheit und Verantwortlichkeit: Drei Grundpositionen

1. Inkompatibilismus 22

2. Kompatibilismus I (freiheitsbejahend) 22

3. Kompatibilismus II (freiheitsverneinend, aber verantwortlichkeitsbejahend)

IV.Die inkompatibilistischen Positionen: Prinzipielle Argumente und Grenzen

1. Determinismus 24

1.1 Quantenmechanik? 26

1.2 Neuronaler, nicht universaler Determinismus $\quad 30$

1.3 Fatalismus? 34

2. Indeterminismus 35

2.1 Die destruktive Strategie 36

2.2 Das „Gründe versus Ursachen"-Argument 39

2.3 Die konstruktive Strategie 51

2.3.1 Akteurskausalität I: die Freiheitslehre Immanuel Kants 51

2.3.2 Kritik 60

2.3.3 Akteurskausalität II: heutige / andere Formen des libertären Inkompatibilismus

3. Resümee zu den inkompatibilistischen Freiheitslehren $\quad 78$

V. Die kompatibilistischen Positionen: Grundlagen und Grenzen 79 1. Das Gehirn-Geist-Problem und seine Bedeutung für die 
Frage der Willensfreiheit $\quad 80$

$\begin{array}{lll}1.1 \text { Identitätstheorien } & 80\end{array}$

1.1.1 Mentale Verursachung? 83

1.1.2 Qualia, oder: Was Mary nicht wissen konnte 87

1.2 Andere Lösungen: Moderate Dualismen - Emergenz?

Supervenienz? 91

1.3 Resümee 95

2. Andershandelnkönnen (PAM) als notwendige Bedingung für Freiheit und Verantwortlichkeit?

Der Kompatibilismus Harry G. Frankfurts 96

2.1 Frankfurts Angriff auf PAM 97

2.2 Autonome Selbstvergewisserung statt Andershandeln können? 102

2.3 Resümee; Überleitung zum Schuldprinzip 104

VI. 20 StGB: zur Legitimation eines vernünftigen

strafrechtlichen Schuldprinzips

1. Zur Auslegung der Norm

2. Zur Legitimation der normativen Zuschreibung von Schuld

2.1 Das sog. subjektive Freiheitsempfinden 118

2.2 Das objektive Fundament des Schuldprinzips $\quad 121$

2.2.1 Der Blick auf die Belange des Täters

2.2.2 Der Blick auf die Bedingungen rechtlicher Ordnung

VII. Resümee: Vorschlag zur Bescheidenheit 